\title{
Effect of the Putative Lithium Mimetic Ebselen on Brain Myo-Inositol, Sleep, and Emotional Processing in Humans
}

\author{
Nisha Singh ${ }^{1,2,6}$, Ann L Sharpley², Uzay E Emir ${ }^{3}$, Charles Masaki ${ }^{2}$, Mohammad M Herzallah ${ }^{4,5}$, Mark A Gluck ${ }^{4}$, \\ Trevor Sharp', Catherine J Harmer', Sridhar R Vasudevan', Philip J Cowen ${ }^{2}$ and Grant C Churchill*,I \\ 'Department of Pharmacology, University of Oxford, Oxford, UK; ²Department of Psychiatry, University of Oxford, Warneford Hospital, Oxford, \\ UK; ${ }^{3}$ The Oxford Centre for Functional MRI of the Brain, Nuffield Department of Clinical Neurosciences, University of Oxford, John Raddliffe \\ Hospital, Oxford, UK; ${ }^{4}$ Center for Molecular and Behavioral Neuroscience, Rutgers, The State University of New Jersey, Newark, NJ, USA; \\ ${ }^{5}$ Palestinian Neuroscience Initiative, Faculty of Medicine, Al-Quds University, Abu Dis, Jerusalem, Palestine
}

\begin{abstract}
Lithium remains the gold standard in treating bipolar disorder but has unwanted toxicity and side effects. We previously reported that ebselen inhibits inositol monophosphatase (IMPase) and exhibits lithium-like effects in animal models through lowering of inositol. Ebselen has been tested in clinical trials for other disorders, enabling us to determine for the first time the effect of a blood-brain barrier-penetrant IMPase inhibitor on human central nervous system (CNS) function. We now report that in a double-blind, placebo-controlled trial with healthy participants, acute oral ebselen reduced brain myo-inositol in the anterior cingulate cortex, consistent with CNS target engagement. Ebselen decreased slow-wave sleep and affected emotional processing by increasing recognition of some emotions, decreasing latency time in the acoustic startle paradigm, and decreasing the reinforcement of rewarding stimuli. In summary, ebselen affects the phosphoinositide cycle and has CNS effects on surrogate markers that may be relevant to the treatment of bipolar disorder that can be tested in future clinical trials.

Neuropsychopharmacology (2016) 4I, I768-1778; doi:I0.1038/npp.2015.343; published online 16 December 2015
\end{abstract}

\section{INTRODUCTION}

Bipolar disorder affects $1-3 \%$ of the world population and is one of the biggest disease burdens in industrialized countries (Lopez et al, 2006). Lithium is the most effective treatment for bipolar disorder, as it controls both mania and depression and decreases suicide (BALANCE investigators and collaborators et al, 2010), but the therapeutic window is small, and it has several unwanted side effects (McKnight et al, 2012), some medically serious. Other drugs such as anticonvulsants and antipsychotics used to treat bipolar disorder are not as effective in stabilizing mood and preventing suicide as lithium (McKnight et al, 2012). Indeed, no agent has been specifically developed as a mood stabilizer for bipolar disorder on the basis of an understanding of the illness or mechanism of action of effective treatments (Conn and Roth, 2008).

Rational design of a mood stabilizer could be pursued based on the mechanism of action of lithium, but its therapeutic

*Correspondence: Dr GC Churchill, Department of Pharmacology, University of Oxford, Mansfield Road, Oxford OXI 3QT, UK, Tel: +44 (0)।865 27| 635, Fax: +44 (0)1865 27| 853,

E-mail: grant.churchill@pharm.ox.ac.uk

${ }^{6}$ Current address: Centre for Neuroimaging Sciences, Box 089, Institute of Psychiatry, Psychology and Neuroscience, De Crespigny Park, London SE5 8AF, UK.

Received 21 August 2015; revised 25 October 2015; accepted II November 2015; accepted article preview online 23 November 2015 target remains unknown (Quiroz et al, 2004). Based on clinically relevant lithium concentrations $(0.6-1.2 \mathrm{mM})$, the two most likely targets are glycogen synthase kinase 3 and inositol monophosphatase (IMPase) (Agam et al, 2009; O'Brien and Klein, 2009). Evidence for and against both targets comes from animal studies employing genetic and pharmacological manipulations (Agam et al, 2009; O'Brien and Klein, 2009). However, as certain animal models are reported to be poor predictors of clinical efficacy (Conn and Roth, 2008; Harmer et al, 2011), both therapeutic mechanism and target validation must be evaluated ultimately in humans. In this regard, surrogate efficacy markers in healthy volunteers are emerging as a promising bridge to efficacy studies (Harmer et al, 2011).

Berridge's 'inositol depletion hypothesis' has provided an attractive mechanistic explanation for the action of lithium and a rationale for testing it by predicting a lowering in myo-inositol levels (Berridge et al, 1989). This hypothesis proposes that lithium normalizes signaling in overactive neurons by inhibiting IMPase and depleting myo-inositol. As inositol is poorly blood-brain barrier penetrant, brain cells depend largely on recycling and de novo synthesis rather than uptake, making the central nervous system (CNS) uniquely sensitive to IMPase inhibition (Berridge et al, 1989). The location of IMPase in inositol metabolism enables it to exert control of several diverging downstream pathways including cytosolic calcium, protein kinase $\mathrm{C}$, protein kinase B, and phosphatidylinositol 3-kinase (Figure 1a). 
a

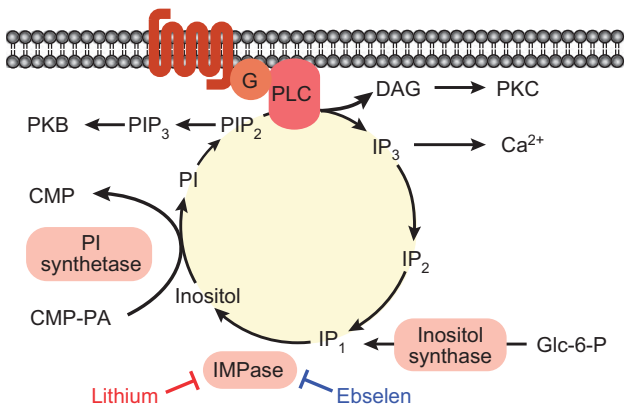

d
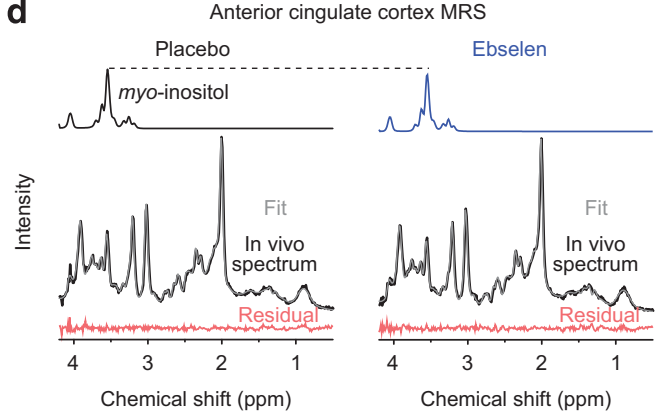

e Anterior cingulate cortex

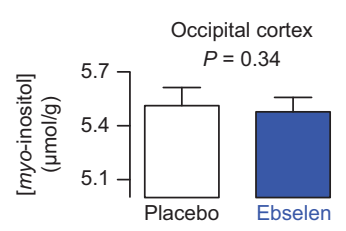

f

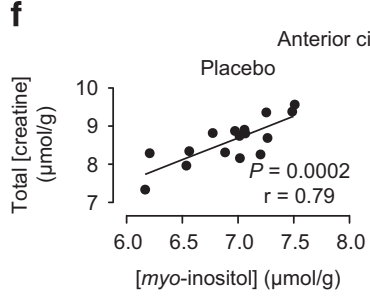

b

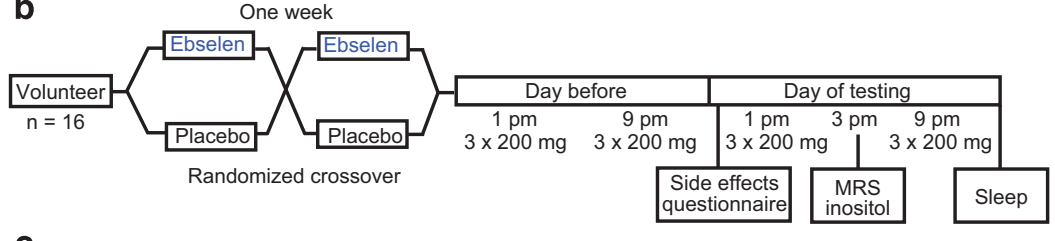

C
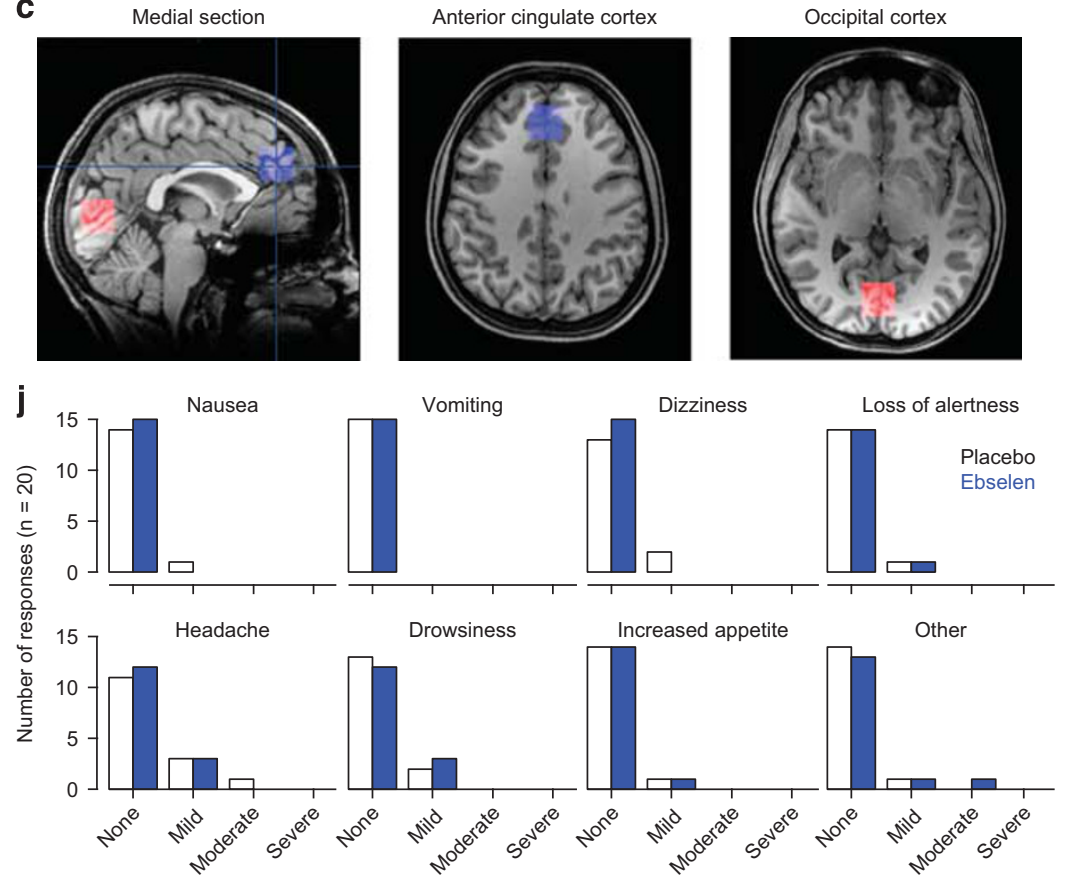

g
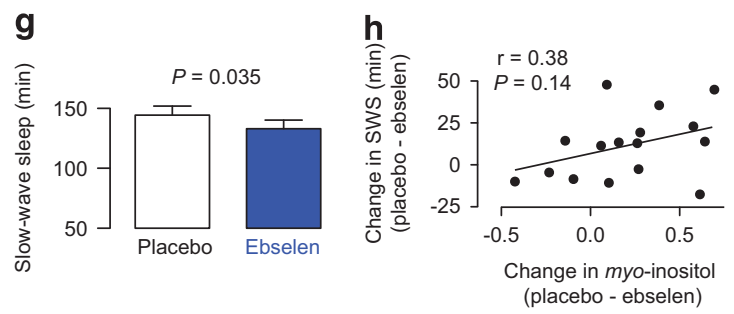

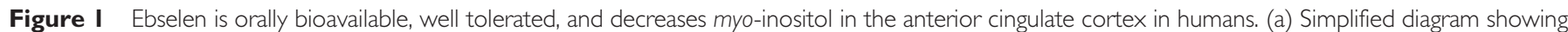

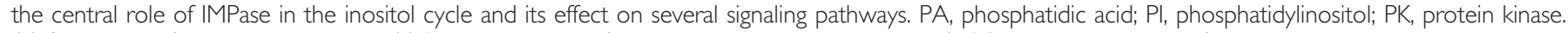

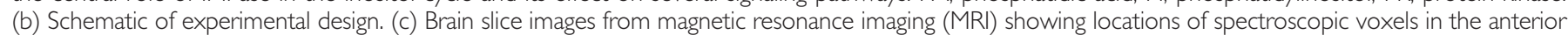

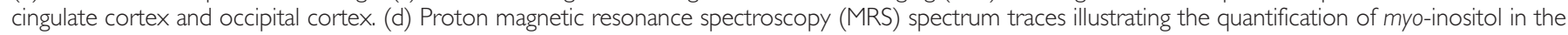

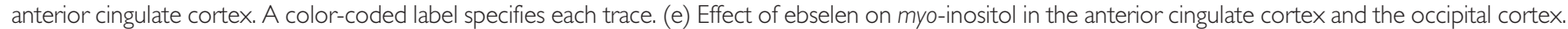

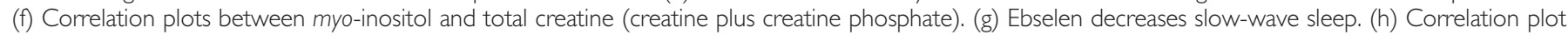

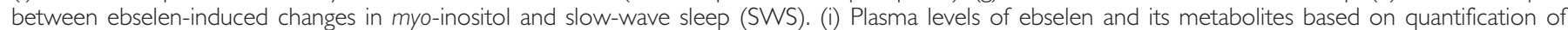

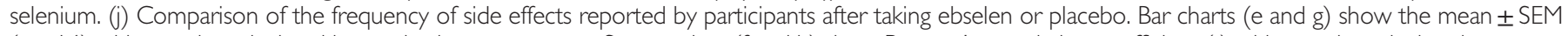

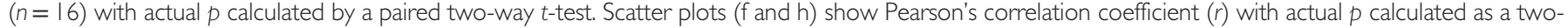
way test $(n=16)$.

The hypothesis has been tested by measuring myo-inositol levels in animals and humans, but with mixed results possibly because of a combination of compensatory changes during the ramp up to pharmaceutical concentrations in the CNS and lithium inhibition of multiple targets (Davanzo et al, 2003; Moore et al, 1999; Patel et al, 2008; Sharma et al, 1992; Silverstone et al, 2005; Silverstone and McGrath, 2009). The only known selective IMPase inhibitor, L-690,330, developed by Merck, Sharpe, and Dohme, affects the inositol cycle in mouse brain but required high doses $(\mathrm{mmol} / \mathrm{kg})$ because of its high polarity and lack of membrane permeability (Atack et al, 1993).
Evaluation of IMPase in humans has not been possible because of the lack of a bioavailable, blood-brain barrier-penetrant inhibitor. Recently, we reported that ebselen inhibited IMPase and caused lithium-like neuropharmacological effects in mice (Singh et al, 2013). Ebselen is a bioavailable antioxidant drug that has been tested in humans for other diseases but has not been marketed (Lynch and Kil, 2009; Parnham and Sies, 2013; Yamaguchi et $a l, 1998)$ and can therefore be 'rescued' for a new disorder (Cavalla, 2009). We have now used ebselen to determine the effect of IMPase inhibition on CNS function in humans. 


\section{MATERIALS AND METHODS}

\section{Study Oversight}

The studies were overseen by the Oxford University Clinical Trials and Research Governance team and sponsored by the University of Oxford. Ethical approval was granted from the National Research Ethics Service Committee South Central. Informed consent was obtained from each participant. A safety-monitoring group collated adverse events.

\section{Study Design and Participants}

The sleep and inositol study involved 16 healthy participants (6 females and 10 males) in a randomized, double-blind, placebo-controlled, crossover design (Figure 1b). Ebselen capsules and identical matching placebo (containing Avicel microcrystalline cellulose) capsules were purchased from Shasun Pharmaceuticals. Ebselen was administered as $3 \times 200 \mathrm{mg}$ capsules. The day before the test day, the first dose was administered (by the participant themselves) at $1300 \mathrm{~h}$ and then again $2 \mathrm{~h}$ before bed. The following morning, within $20 \mathrm{~min}$ of waking, participants completed the Leeds Sleep Evaluation Questionnaire (Parrott and Hindmarch, 1980) and side-effects profile using a subjective 4-point rating scale. Participants administered the third dose at $1300 \mathrm{~h}$ and were scanned at the Oxford Centre for Clinical Magnetic Resonance Research (OCMR), University of Oxford, at $1500 \mathrm{~h}$. A blood sample was collected at $1615 \mathrm{~h}$ and the participants were then fitted with the ambulatory Embla Titanium sleep recorder (Natus Neurology, Middleton, WI) and returned home to sleep as usual. The final dose was administered $2 \mathrm{~h}$ before sleeping. The following morning, participants completed the Leeds Sleep Evaluation Questionnaire and side-effects profile. The study was repeated a week later with the alternative treatment. On completion, participants were asked to guess on which occasion they had taken the ebselen and placebo.

The emotional processing study involved 40 healthy participants, 20 per group, in a randomized, double-blind, placebo-controlled, parallel-group design (Figure 2b). The group demographics and results from baseline screening questionnaires are given in Table 1 . The day before the test day, $\sim 15$ min before the administration of the first dose of ebselen/placebo $(3 \times 200 \mathrm{mg})$ at $\sim 0930 \mathrm{~h}$, participants were asked to complete a set of baseline questionnaires: positive and negative affective schedule (Watson et al, 1988), the Befindlichkeit mood and energy questionnaire (Von Zerssen, 1986), the Beck depression inventory, visual analog scales (Bond and Lader, 1974), and a state-anxiety questionnaire (Spielberger et al, 1970). Participants were asked to take the second dose $(3 \times 200 \mathrm{mg})$ at $\sim 2130 \mathrm{~h}$. On the test day, participants took the final dose $(3 \times 200 \mathrm{mg})$ at $0915 \mathrm{~h}$ in the department. At $1115 \mathrm{~h}$, the participants again completed the above questionnaires as well as one for side effects (a 4-point rating scale) to reveal any drug-related effects. Testing began at $1130 \mathrm{~h}$ and was carried out in the same order and by the same researcher, in all cases.

\section{Participant Exclusion Criteria}

The exclusion criteria were as follows: history of any axis I psychiatric disorder (determined at screening using the
Standard Clinical Interview for Diagnostic and Statistical Manual for Mental Health Disorders-fourth edition); pregnancy or lactation; current usage of any other prescription medication, except the oral contraceptive; any other medical condition; and heavy smokers. Specific exclusion criteria for the sleep and inositol study included fulfilling safety requirements to allow admittance to the scanner, any current or past sleep disorder and claustrophobia, and, for the emotional processing study, prior exposure to the battery of psychological tests, dyslexia, and poor spoken or written English. Participants were asked to abstain from alcohol while participating in the study.

\section{Emotional Processing Tasks}

Full details of the tasks are provided in the Supplementary Information Subjects and Methods. The first task was the Auditory Verbal Learning Task (Rey, 1964), interspersed with the Reward and Punishment Learning Task (described in detail below). This was followed by the Emotional Testing Battery conducted as described previously (Harmer et al, 2009; Murphy et al, 2008) that constituted the Facial Emotion Recognition Task, the Emotional Categorization, the Dot Probe Task, the Emotional Recall, and the Emotional Recognition Task. The final task was the Emotion Potentiated Startle Task (Lang et al, 1993). The testing was usually complete in $\sim 2 \mathrm{~h}$ and participants were given breaks at certain designated times. At the end of the study the participants were asked to report whether they thought they were on placebo or ebselen.

\section{Reward and Punishment Learning Task}

This computer-based cognitive task is a within-subject assessment of probabilistic learning from negative and positive feedback. It is divided into two separate reward and punishment conditions of 100 trials each. Every participant undertakes both conditions, and the order of presentation of conditions is randomized. In each condition, the 100 trials are divided into blocks of 25 trials (4 blocks of 25 trials per condition). In the reward condition, the person gains points for making the most winning choice, and in the punishment condition, the person does not lose points for making the least losing choice. On each trial, the subject is presented with four decks of cards, each with probabilities of $50 \%, 60 \%, 70 \%$, and $80 \%$, respectively, of gaining reward (reward condition) or of generating punishment (punishment condition). One deck of the four will have the most optimal deck of cards. This deck will be the most winning in the reward condition, and the least losing in the punishment condition. Three other nonoptimal decks of cards were presented with the optimal deck. In each trial, the participant is asked to choose one of the four decks with the expectation that over time, the participant will learn which of the four decks is the most optimal deck.

In the reward condition, the participant starts with a score of 0 and is asked to choose the 'best' deck, which in this case is the deck with $80 \%$ probability of gain. The choice of the optimal deck yields in positive feedback by the addition of 10 points, otherwise nothing. The participant is expected to continue selecting the optimal stimulus after learning it, despite the lack of positive feedback in some of the trials. 
a

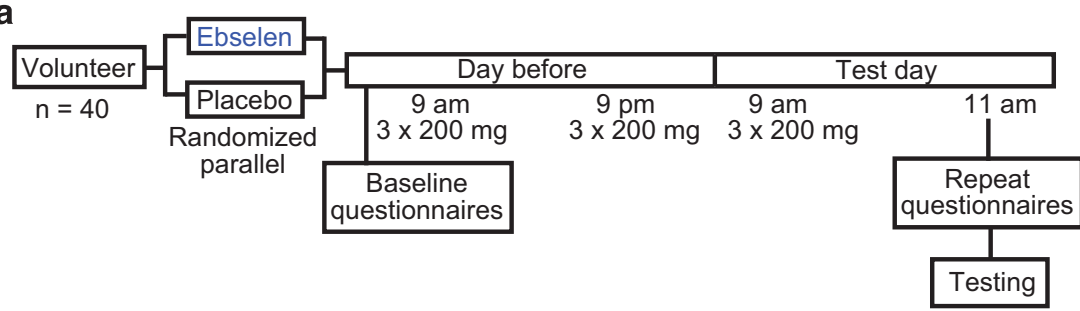

b

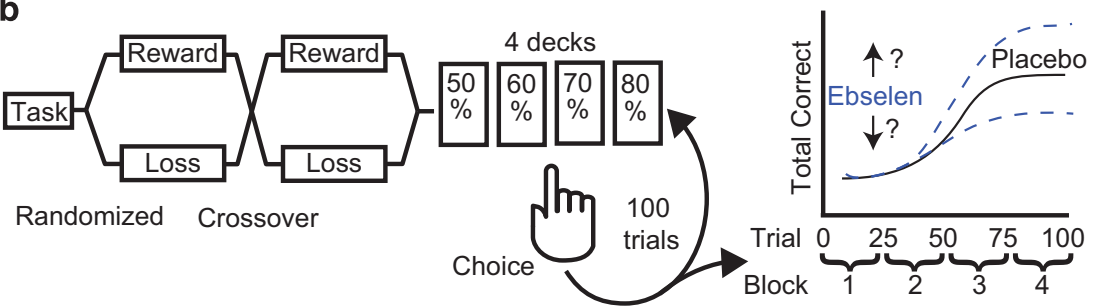

C Reward \& loss paradigms together

d Reward paradigm Block $x$ Treatment Interaction

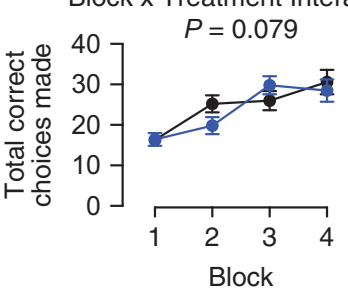

Ebselen

Placebo e

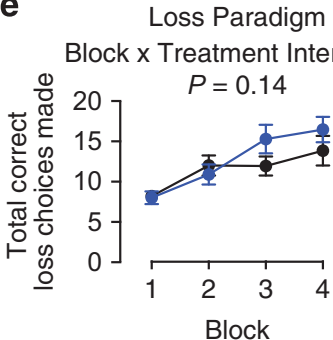

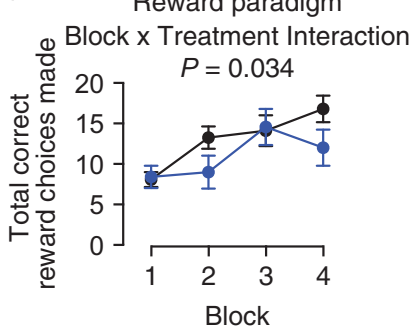

f

Learning paradigm

Paradigm $\times$ Treatment Interaction

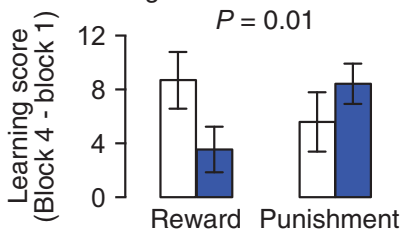

Figure 2 Ebselen affects learning influenced by either reward or punishment. (a) Schematic of experimental design. (b) Schematic of the reward and punishment learning task. The task involves picking one card at a time from four decks with two conditions, 'pick the best deck' or 'avoid the worst deck', and through trial and error the participant learns which of the four decks of virtual cards provides the most points in a paradigm of reward or punishment. Ebselen could increase or decrease learning relative to the placebo learning response. (c-e) Effect of ebselen on total correct choices made over time under conditions of both reward and punishment (c), as well as under the reward (d) and punishment (e) condition separately. ( $f$ ) Effect of ebselen on total learning. Statistical comparisons were made with a two-way repeated measures analysis of variance with actual $p$ shown $(n=20)$

In the punishment condition, the participant is asked to avoid the 'worst deck' or the deck with the highest probability of punishment. They start with a score of 1000 and if the deck chosen yields punishment, they lose points. The optimal deck in this condition is the $50 \%$ probability of loss. Again, the participant is expected to continue selecting the optimal deck after learning it, despite the negative feedback in some of the trials.

The optimal choices for reward (best deck $=80 \%$ probability of gain) and for loss (best deck $=50 \%$ probability of loss) are rewarded one point each and added to calculate the total optimal choice accuracy. Learning is calculated by subtracting the total optimal choices made in block 1 from the total optimal choices made in block 4 for each condition.

\section{Sleep Architecture}

On each study night, polysomnograms were recorded as each participant slept at home, using the Embla titanium recording system (Natus Neurology Incorporated). Participants retired and rose at their usual time, and this was kept constant for all study nights and all preceding nights. The industry standard montage was used that comprised six electroencephalogram channels (C3-M2, C4-M1, O1-M2, O2-M1, F3-M2, and F4-M1), two electrooculogram channels (E1-M2 and E2-M2), and submentalis electromyogram channels using three electrodes. Polysomnograms were staged in 30-s epochs using the Embla RemLogic sleep diagnostic software (Natus Neurology). RemLogic adheres to the American Academy of Sleep Medicine scoring criteria. In 
Table I Demographics of the Two Participant Groups (Placebo and Ebselen) and the Information from the Screening Questionnaires

\begin{tabular}{lccc}
\hline $\begin{array}{l}\text { Screening } \\
\text { questionnaires }\end{array}$ & $\begin{array}{c}\text { Placebo group, } \\
\text { mean } \pm \text { SEM (N) }\end{array}$ & $\begin{array}{c}\text { Ebselen group, } \\
\text { mean } \pm \text { SEM (N) }\end{array}$ & P-value \\
\hline Age & $21.95 \pm 0.70(20)$ & $23.25 \pm 0.96(20)$ & 0.28 \\
Sex & 8 Males, I2 females & 7 Males, I3 females & - \\
BMI & $21.95 \pm 0.36(20)$ & $22.20 \pm 0.45(20)$ & 0.66 \\
National adult & $122.9 \pm 0.49(20)$ & $123.1 \pm 0.42(19)$ & 0.71 \\
reading test & & & \\
Trait anxiety & $31.80 \pm 1.13(20)$ & $33.65 \pm 1.52(20)$ & 0.34 \\
State anxiety & $50.10 \pm 0.61(20)$ & $50.32 \pm 0.52(19)$ & 0.79 \\
Eysenck personality & $32.25 \pm 1.19(20)$ & $31.05 \pm 1.47(20)$ & 0.53 \\
questionnaire & & & \\
Positive and negative & & & \\
affect schedule & & & \\
$\quad$ Positive & $28.40 \pm 1.50(20)$ & $28.75 \pm 1.78(20)$ & 0.88 \\
$\quad$ Negative & $12.60 \pm 0.67$ & $11.95 \pm 0.44$ & 0.42 \\
Befindlichkeit & & & \\
questionnaire & & & \\
$\quad$ Mood & $17.15 \pm 3.07(20)$ & $13.25 \pm 2.17(20)$ & 0.31 \\
$\quad$ Energy & $5.90 \pm 1.13(20)$ & $4.35 \pm 1.16(20)$ & 0.35 \\
Beck depression & $3.34 \pm 0.68(19)$ & $4.20 \pm 0.89(20)$ & 0.47 \\
inventory & & & \\
\hline
\end{tabular}

addition, an experienced sleep physiologist who was blind to the treatment status analyzed the polysomnograms.

\section{Proton Magnetic Resonance Spectroscopy}

Spectra were measured using the Siemens Trio 3-Tesla whole body MRI scanner and a 32-channel coil at the OCMR (University of Oxford). A high-resolution T1-weighted MP RAGE (Brant-Zawadzki et al, 1992) image was acquired for accurate MRS voxel placement and subsequent structural analyses $\left(\mathrm{TR}=2040 \mathrm{~ms}, \mathrm{TE}=4.7 \mathrm{~ms}\right.$, flip angle $=8^{\circ}, 192$ transverse slices, 1-mm isotropic voxels). B0 shimming was achieved using a GRESHIM (Shah et al, 2009), available as a work-in-progress package on the Siemens system. Spectra were measured with a semiadiabatic localization by adiabatic selective refocusing (SEMI-LASER) sequence (TE $=28 \mathrm{~ms}$; $\mathrm{TR}=4 \mathrm{~s} ; 64$ averages) with variable power radiofrequency pulses with optimized relaxation delays (VAPOR) water suppression and outer volume saturation (Deelchand et al, 2014). Unsuppressed water spectra acquired from the same voxel were used to remove residual eddy current effects and to reconstruct the phased array spectra (Natt et al, 2005). Data were acquired in single-shot mode, that is, a single free induction decay was saved separately. Single-shot spectra were frequency and phase corrected before averaging over 64 scans.

Metabolites were quantified with LCModel (Provencher, 1993) using the unsuppressed water signal as reference. T1-weighted structural images were brain extracted and tissue-type segmented using the Brain Extraction Tool of FMRIB Software Library (FSL) (Smith, 2002) and Automated Segmentation Tool of FMRIB (Zhang et al, 2011). The percentage of gray matter, white matter, and cerebrospinal fluid within the MRS voxel was calculated from the resulting images and used to correct metabolite concentrations for cerebrospinal fluid fraction.

\section{Statistics}

We used two-tailed $t$-test to compare two means and analysis of variance to compare more than two means followed by Bonferroni post hoc test or $t$-tests corrected for multiple comparisons. Where possible, actual $p$-values are provided rather than a cutoff to declare significance or not. For data in which an underlying relationship was evident, we used regression and global nonlinear curve fitting as this provides more statistical power (Motulsky and Christopoulos, 2003).

\section{RESULTS}

\section{Ebselen Reduces Myo-Inositol in the Anterior Cingulate Cortex}

In studies using mice, ebselen decreased myo-inositol in brain extracts (Singh et al, 2013). To determine whether ebselen inhibits IMPase in vivo in humans, we used proton magnetic resonance spectroscopy (MRS) to quantify myoinositol in healthy participants. In a double-blind, randomized crossover experimental design (Figure $1 \mathrm{~b}$ and Table 1), ebselen reduced myo-inositol in the anterior cingulate cortex but not the occipital cortex (Figure 1c-e). In addition to myo-inositol, we also used MRS to quantify several other metabolites, and although some trends were observed such as an increase in combined glutamine and glutamate and a decrease in ascorbate, creatine, and choline, no changes were significant after correction for multiple comparisons (Supplementary Figure S1). Total creatine (creatine plus phosphocreatine) levels positively correlated with myo-inositol in both the placebo and ebselen groups (Figure 1f).

\section{Ebselen Decreases Slow-Wave Sleep}

Many psychotropic drugs used to treat mood disorders, including lithium and antidepressants, have defined effects on sleep (Friston et al, 1989; Sharpley et al, 1994). Lithium extends slow-wave sleep, also termed stage N3 sleep, through a mechanism thought to involve the $5-\mathrm{HT}_{2}$ receptors (Friston et al, 1989), possibly through inhibition of IMPase. To determine the effects of ebselen on sleep architecture, we monitored sleep in the same healthy participants as in the MRS study (Figure 1b). Ebselen decreased slow-wave sleep (Figure 1g) by reducing the number of slow-wave sleep episodes $(p=0.058)$ rather than the duration of each slow-wave sleep episode (Supplementary Table S1). Ebselen produced no other significant effects on sleep (Supplementary Table S1), and the decrease in slow-wave sleep did not affect either the total sleep time (Supplementary Table S1) or total sleep quality, as reported by the Leeds Sleep Evaluation Questionnaire (Parrott and Hindmarch, 1980) (Supplementary Figure S2). No correlation between the effect of ebselen on slow-wave sleep and myo-inositol was observed $(r=0.38, p=0.14$; Figure $1 \mathrm{~h})$. Taken together, these data show that ebselen decreases slow-wave sleep. 


\section{Oral Ebselen is Bioavailable and Well Tolerated}

Plasma selenium concentrations have been shown to correlate with plasma levels of ebselen (Fischer et al, 1988; Lynch and Kil, 2009). Using selenium as a surrogate measure of ebselen and its metabolites, we found higher selenium in plasma of the participants taking ebselen than placebo (Figure 1i), demonstrating both compliance with dosing and the bioavailability of oral ebselen in humans, as reported previously (Fischer et al, 1989; Lynch and Kil, 2009; Yamaguchi et al, 1998). In our parallel arm experimental design (Figure $1 \mathrm{~b}$ ), participants who received ebselen then placebo 1 week later would have a predicted remaining amount of ebselen of $0.006 \%$ of the initial dose calculated from a reduction of $\sim 14$ half-lives (Lynch and Kil, 2009), which should be a sufficient washout time. Plasma selenium levels correlated with the magnitude of decrease in slowwave sleep $(p=0.017 ; r=0.36)$, but not with myo-inositol levels $(p=0.70)$. The participants consistently reported the same low number and low intensity of side effects with ebselen as the placebo (Figure $1 \mathrm{j}$ ), consistent with the acceptable tolerability of ebselen as reported previously (Lynch and Kil, 2009; Parnham and Sies, 2013; Yamaguchi et al, 1998). Although mood questionnaires were administered, ebselen did not induce any changes relative to the placebo (Table 1), but this is not surprising because the dose was acute and even validated antidepressants taken acutely do not alter mood ratings (Harmer et al, 2011). Moreover, participants were healthy volunteers with low levels of negative affect and therefore may be relatively insensitive to change in subjective state.

\section{Ebselen Decreases Reward Stimulus Reinforcement}

Many neuropsychiatric disorders, including mania, are associated with an increase in poor decision making because of increased impulsivity and a hypersensitivity to reward (Whitton et al, 2015). As probing such functions invokes tasks that implicitly measure optimal choices made over time, we used a randomized, parallel-arm, experimental design (Figure 2a). To determine the effect of ebselen on decision making and learning, we used a task that tests the ability to distinguish between stimuli (decks of cards) with different probabilistic values of gain or loss, and the influence of learning from positive and negative reinforcement (Figure 2b). The task is divided into two conditions: the reward condition and the punishment condition. Each time the person has to chose one out of four decks of cards where they are likely to gain the most, that is, add points in the reward paradigm or not lose points in the punishment paradigm.

Ebselen had no overall discernable effect on the total correct choices made, when both the reward and loss paradigms were combined together (Figure 2c). However, when the correct choices made for rewarding stimulus and punishing stimulus were analyzed separately, ebselen significantly decreased the total correct reward choices made $(p=0.034$; Figure $2 \mathrm{~d})$, although no significant increase in total correct loss choices made $(p=0.14$; Figure $2 \mathrm{e})$ was observed. This interaction was further corroborated through a learning measure that showed a significant interaction between the reward and loss paradigms and ebselen $(p=0.01)$, whereby ebselen decreases reward reinforcement and increases punishment reinforcement $(p=0.01$; Figure 2f). Taken together, these data show that ebselen decreases learning through reward reinforcement.

\section{Ebselen Increases Recognition of Disgust and Happiness in Emotional Processing Tasks}

In mood disorders there are alterations in neural circuits regulating emotional processing, emotional regulation, and reward processing (Phillips and Swartz, 2014). Moreover, many psychotropic drugs affect tasks involving these processes (Phillips and Swartz, 2014), and certain emotional processing tasks in healthy participants respond to drugs in a manner that is predictive of clinical effect, suggesting such tasks might provide insight into the ultimate efficacy of new drugs (Harmer et al, 2011). To determine whether ebselen alters emotional processing, we used a battery of computerized tasks designed to tap basic emotional processing and mood responses to positive and negative emotional material. As the tasks have significant learning effects we used naive participants in a randomized parallel experimental design (Figure 2a).

In the facial emotion recognition task, participants are shown randomized pictures of varying degrees (40 for each emotion, 4 per degree) of the emotions anger, disgust, happiness, fear, sadness, and surprise, as well as 10 neutral faces (Figure 3a). Participants given ebselen showed a significant difference in the way they perceived some of these emotional faces $(p=0.045$; Figure $3 b)$. Ebselen did not affect miscalculations $(p=0.10)$ or sensitivity $(p=0.078$; Figure $3 b)$. Looking at the recognition of faces over different intensities, clear trends were evident with degrees of emotion (Figure 3c). As statistical power can be gained by fitting data to relationships that are not accounted for in analysis of variance, we applied a curve-fitting analysis using a fourparameter logistic equation (Motulsky and Christopoulos, 2003). Based on this analysis ebselen increased the recognition of anger $(p=0.018)$, disgust $(p<0.0001)$, and happiness $(p=0.003)$, but only disgust and happiness are deemed significant if a Bonferroni correction for multiple comparisons $(p<0.008)$ is applied. No differences were found in the recognition of sadness, fear, and surprise between the two groups (Figure $3 \mathrm{~b}$ and $\mathrm{c}$ ). Importantly, there was no difference in reaction time observed between the participants on placebo and those on ebselen (Figure 3b). Taken together, these results demonstrate that ebselen affected emotional processes in healthy participants. Ebselen did not affect performance in the Dot Probe (attentional bias), the emotional memory tasks (Supplementary Figure S3), or the Auditory Verbal Learning Task (Supplementary Figure S4).

\section{Ebselen Decreases Latency in the Emotion Potentiated Acoustic Startle Task}

Another way of investigating emotional processing is through the effect of emotion on the startle response, one of the most basic and primitive sensory responses to threatening external stimuli (Perry, 1999). Startle is largely modulated by the brain stem, though the emotional modulation might involve the limbic areas (Perry, 1999). 
a

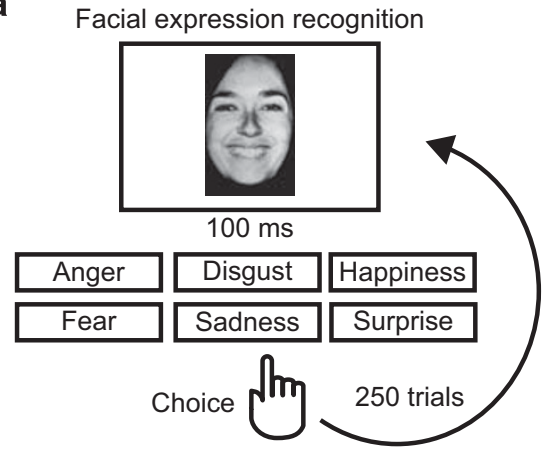

Examples

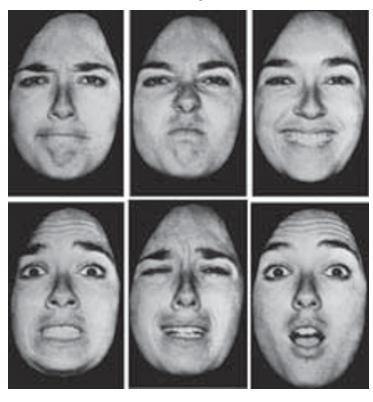

b
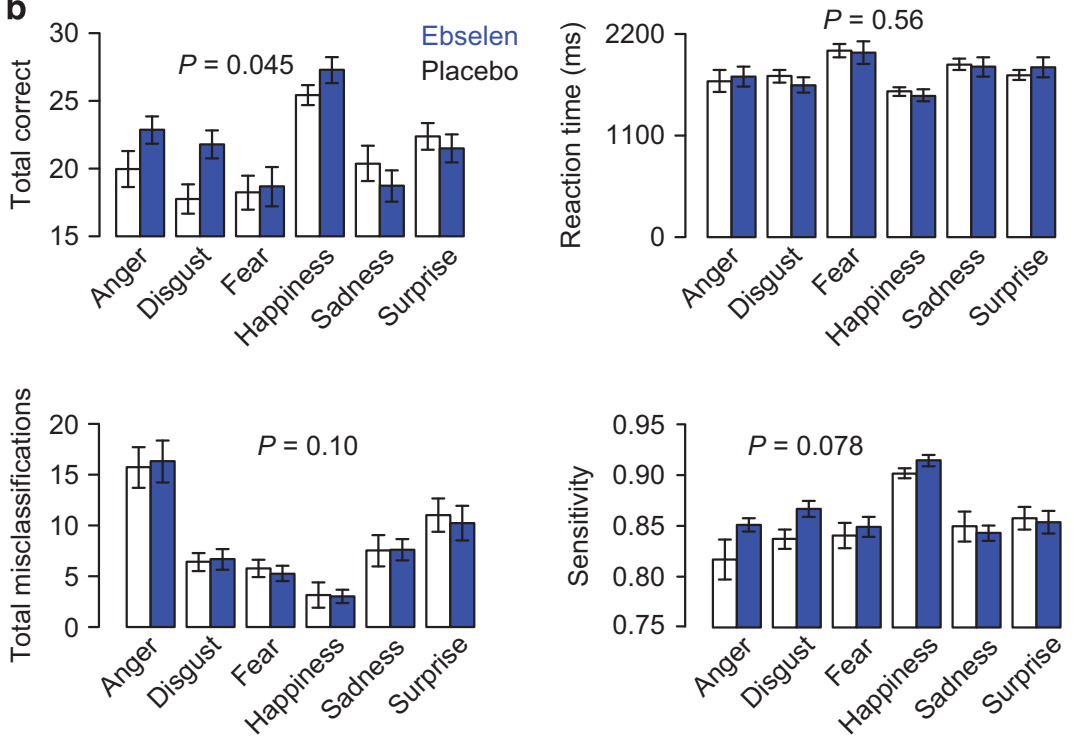
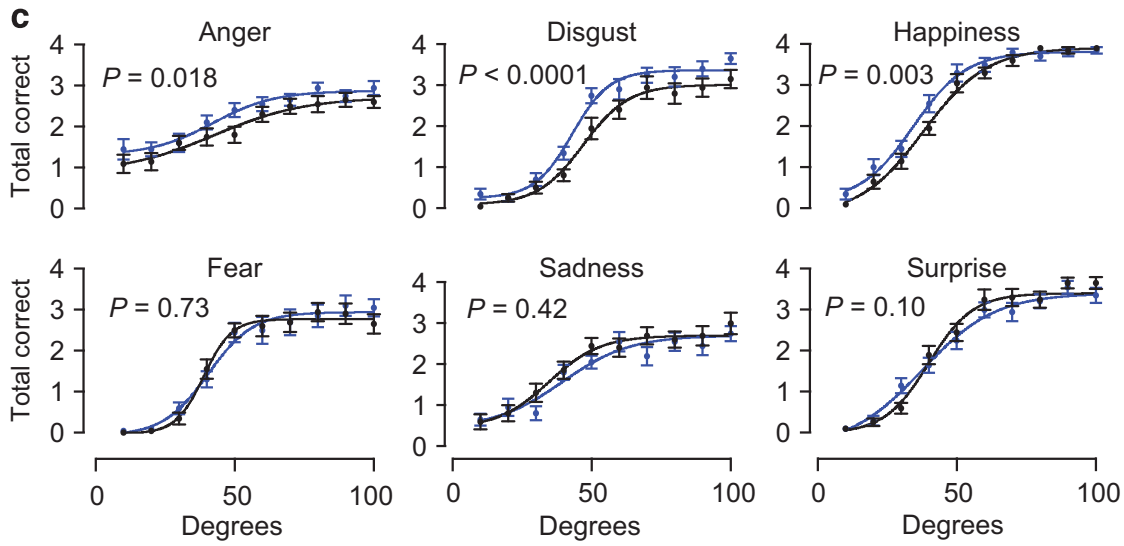

Figure 3 Ebselen increases the recognition of certain emotions but not others in facial expressions. (a) Schematic of the facial emotional expression recognition task. (b) Effect of ebselen on parameters related to the ability to recognize facial emotion. (c) Effect of ebselen on the ability to correctly recognize varying degrees of facial emotional expression. All data are plotted as mean \pm SEM with $n=20$. Statistical comparisons were performed with a two-way repeated measures analysis of variance. Statistical comparisons for (c) were performed by fitting a four-parameter equation (top, bottom, half-maximum and slope) and comparing whether the data sets were better fit with a common curve or independent curves. Actual p-values are shown in the panels.

To determine whether ebselen affected an emotional potentiated startle response, we monitored the effect of viewing pictures that elicit positive, negative, or neutral emotions on the amplitude and latency of the eye-blink component of the startle response (Figure 4a) (Lang et al, 1993). The participants and study design were the same as in the reward-loss and emotional processing tasks (Figure 2a). Ebselen did not affect the amplitude of response $(p=0.51$; Figure $4 \mathrm{~b}$ ). The valence of the emotion (pleasant, neutral, and unpleasant) affected the amplitude of the response $(p=0.009$; Figure $4 \mathrm{~b})$, consistent with previous reports (Murphy et al, 2008), and validates the experimental setup. 

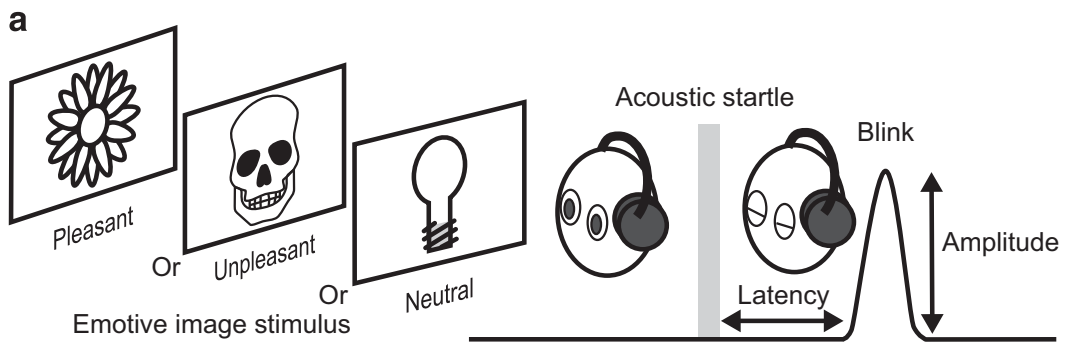

Reflex trace

b
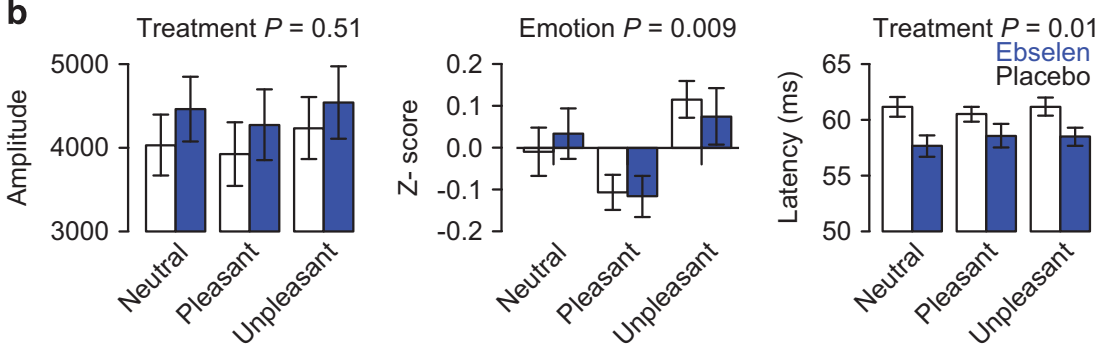

Figure 4 Ebselen decreases the latency in the emotion potentiated startle task. (a) Schematic of the startle task. An emotional image shown for $13 \mathrm{~s}$ modifies the latency and amplitude of an eye blink response elicited by an acoustic startle ( $100 \mathrm{~dB}, 100 \mathrm{~ms})$. (b) Effect of ebselen on the emotionally modified startle reflex. Statistical comparisons were performed with a two-way analysis of variance with the p-values for interaction, treatment, and emotion, respectively: Z-score: $0.08 \mathrm{I}, 0.72$, and 0.009; amplitude: $0.79,0.5 \mathrm{I}$, and $0.01 \mathrm{I}$; and latency: 0.44, 0.0 I, and 0.77. Each graph is labeled with the most salient p-value for its parameter.

Interestingly, ebselen decreased the latency of response $(p=0.012$; Figure $4 \mathrm{~b})$ to the acoustic stimulus and the decrease in latency associated with ebselen was independent of the emotional valence of the picture (treatment $\times$ emotion interaction, $p=0.443$ ).

\section{DISCUSSION}

We conducted an experimental medicine study of ebselen in humans with two interrelated goals: first, to determine whether it is warranted to progress the development of ebselen as a possible treatment for bipolar disorder by determining the effect of ebselen on CNS function, and, second, to gain insight into the mechanism of action of ebselen as a putative lithium mimetic. With regard to our first goal, ebselen is well tolerated and exhibited effects on emotional processing, thereby providing a validation step between animal models and trials for clinical efficacy, justifying continued study of ebselen as a potential treatment for bipolar disorder. Importantly, with regard to side effects and toxicity, none have been reported to date for ebselen at the administered doses (Lynch and Kil, 2009; Parnham and Sies, 2013; Yamaguchi et al, 1998), in marked contrast to lithium (McKnight et al, 2012). With regard to our second goal, the data are consistent with ebselen reducing myoinositol in vivo, thereby indicating target engagement with IMPase.

We identified ebselen through a drug repurposing or rescue approach in which we screened for IMPase inhibitors (Singh et al, 2013). Underlying this approach is that all small molecules exhibit polypharmacology, meaning they affect multiple targets. Ebselen's polypharmacology (Parnham and Sies, 2013) is a double-edged sword with regard to our goals. As a possible treatment for bipolar disorder, ebselen's polypharmacology (Parnham and Sies, 2013) is desired as it inhibits two targets shared with lithium and suggested to be therapeutically relevant including IMPase (Berridge et al, 1989; Quiroz et al, 2004) and protein kinase C (Zarate and Manji, 2009). Moreover, ebselen is an antioxidant, inhibits several of the proinflammatory enzymes, and, like lithium, is a neuroprotectant (Parnham and Sies, 2013). In terms of a novel drug for treating bipolar disorder, the most important things are safety and efficacy. Safety seems excellent based on all animal and human studies to date including the current one. Efficacy for bipolar disorder can be tested, and our results show that this is worthwhile as ebselen has effects in emotional processing tasks that are surrogate markers for efficacy (Harmer et al, 2011).

With regard to our second goal of gaining insight into the therapeutic target and mechanism of lithium, ebselen's polypharmacology complicates definitive conclusions regarding mechanism. All drugs have multiple targets, but insight can be gained by analyzing targets that are shared or unique. Ebselen is $\sim 20$-fold selective for inhibition of IMPase over glycogen synthase kinase-3 $\beta$ (Singh et al, 2013). The main contenders for the therapeutic target of lithium are glycogen synthase kinase- $3 \beta$ and IMPase (Quiroz et al, 2004). As lithium and ebselen affect several targets but IMPase is the known shared target, the most parsimonious explanation for similar effects of lithium and ebselen is inhibition of IMPase. Moreover, using similar logic, it is likely that the side effects and acute toxicity of lithium (McKnight et al, 2012) and its effects on slow-wave sleep (Friston et al, 1989; Sharpley et al, 1994) originate not through IMPase inhibition but through one of its other targets (Quiroz et al, 2004).

That we observed changes in myo-inositol in the anterior cingulate cortex but not the occipital cortex is consistent with the relevance of these regions with regard to bipolar disorder 
and changes in these regions in response to lithium (Davanzo et al, 2003; Moore et al, 1999; Sharma et al, 1992). Indeed, in studies with lithium where changes in myoinositol were observed, changes were in the frontal lobes, which are associated with emotional regulation and linked to mood disorders, and not in the occipital, parietal, or temporal regions (Moore et al, 1999). Indeed, this is why we chose to make our measurements in these two regions. As myo-inositol decreases precede and may be predictive of clinical improvement in bipolar disorder (Davanzo et al, 2003; Moore et al, 1999), ebselen may have clinical utility. In comparison, previous studies with lithium in which myoinositol levels were measured in humans, both in bipolar patients and healthy participants, have yielded mixed results (Davanzo et al, 2003; Moore et al, 1999; Patel et al, 2008; Sharma et al, 1992; Silverstone et al, 2005; Silverstone and McGrath, 2009). Ebselen may have a more immediate and pronounced effect compared with lithium, as it does not have to be slowly titrated to achieve therapeutic concentrations while avoiding toxicity. In addition, we expressed myoinositol in absolute terms (standardized to cerebrospinal fluid fraction in the voxel), avoiding the influence of possible changes in other metabolites such as creatine that can change with treatment (Silverstone et al, 2005; Silverstone and McGrath, 2009). The magnitude of the decrease in myoinositol is modest, but steady-state levels might equate to much larger changes in actual metabolic flux. In addition, although we tested a very specific hypothesis relating to whether ebselen was inhibiting IMPase and causing a reduction in brain myo-inositol, alternative explanations exist and could be tested in future experiments such as ebselen inducing a change in kidney function that affected plasma osmolality that would then result in a change in brain myo-inositol where it functions as an osmolyte (Häussinger et al, 1994).

A well-known difficulty in screening novel drugs for their effects on mood is that even with drugs of validated efficacy, it can take several weeks for the effects to manifest (Harmer et al, 2011). This has stimulated an interest in identifying surrogate markers that would show detectable and quantifiable changes after an acute dosing. Even if no mechanistic explanation exists, a reliable surrogate marker can serve as a valuable step in screening compounds, as it would be fast and inexpensive relative to a clinical trial designed to probe efficacy in a given disorder. A particularly promising area for surrogate markers that respond to drugs with validated longterm efficacy in the treatment of mood disorder but show a rapid, reproducible, and quantifiable response is emotional processing (Harmer et al, 2004, 2009, 2011; Murphy et al, 2008; Rock et al, 2010). These tests are designed to tap into the neuronal circuits postulated to be altered in patients with mood disorder and manifest as negative emotional bias in tasks such as recognizing the mood in faces (Harmer et al, 2011). For example, depressed patients demonstrate negative emotional bias in the facial recognition task by being less likely to recognize happiness and more likely to recognize sadness. The utility of these tasks is potentially great because even in healthy subjects, drugs can predictably alter emotional bias and which correlates with the known longterm efficacy of the drugs in treating depression (Harmer et al, 2011).
Ebselen affected emotional processing in healthy participants. The effect sizes are in the moderate range based on Cohen's $d$ and consistent with the effect size induced by other drugs used in the treatment of mood disorder (Harmer et al, 2004, 2009, 2011; Murphy et al, 2008; Rock et al, 2010). These results provide good evidence to suggest that ebselen is having effects on pathways known to be modulated by drugs currently in use to treat mood disorders. Several of the observed responses to ebselen can be taken as promising in relation to using it in the treatment of bipolar disorder. For example, that ebselen decreases learning through reward reinforcement may be relevant to bipolar disorder given that patients, especially in the manic or hypomanic phases, have an increased tendency to make impulsive decisions, gamble, and are hyperresponsive to rewarding stimuli (Whitton et al, 2015). Ebselen may increase the caution with which a choice is being made that might reduce reckless behavior in bipolar patients or patients with increased impulsivity. Similarly, ebselen increases the recognition of happy faces (positive expression) as well as disgusted faces (negative expression). Antidepressants also show significant effects in this task, but typically increase the relative processing of positive rather than negative facial expressions (Harmer et al, 2004). The effect of ebselen to increase recognition of disgust is intriguing because previous studies with the same task revealed the same trend in medicated euthymic bipolar patients (Harmer et al, 2002). Moreover, untreated students at risk of mood disorders are less able to identify 'disgust' accurately (Rock et al, 2010).

A crisis exists in drug development for mental health with industry abandoning research and development on psychiatric disorders because of the lack of both mechanistic understanding and validated targets (Conn and Roth, 2008). This has opened opportunities for academics to pursue strategies such as finding new uses for old drugs termed repurposing for marketed drugs or rescuing for abandoned drugs (Cavalla, 2009; Conn and Roth, 2008). The major promise of these strategies is that they can facilitate the rapid translation of results into humans because the compounds have cleared the early hurdles of drug development. However, development of a marketed drug for a new disease is often prevented by commercial, regulatory, and reimbursement challenges (Cavalla, 2009). In contrast, development of a nonmarketed drug like ebselen for bipolar disorder is more attractive because of enforceable market exclusivity based on a 'use' patent (Cavalla, 2009).

\section{CONCLUSIONS}

Ebselen decreases myo-inositol in brain regions associated with emotional processing consistent with inhibition of IMPase as proposed by the inositol depletion hypothesis (Berridge et al, 1989). These results highlight the potential for developing more selective inhibitors of IMPase or other targets in the phosphoinositol cycle. Ebselen affected performance in several emotional processing tasks. As these tasks are surrogate markers for affective disorders and correlate with efficacy of known mood-modulating drugs (Harmer et al, 2011), it is warranted to test ebselen for efficacy in bipolar disorder. 


\section{FUNDING AND DISCLOSURE}

This work was supported by a Follow-on Fund grant $(\mathrm{BB} / \mathrm{J} 021547 / 1)$ from the Biotechnology and Biological Science and the John Fell Oxford University Press Research Fund, University of Oxford. Grant C Churchill, Sridhar R Vasudevan, and Nisha Singh hold a method-of-use patent (WO2012107735 A2) for ebselen in the treatment of bipolar disorder. The other authors declare no conflict of interest.

\section{ACKNOWLEDGMENTS}

We thank Steven Knight (Universality of Oxford Centre for Clinical Magnetic Resonance) for expert technical assistance with the MRS.

\section{AUTHOR CONTRIBUTIONS}

GCC, SRV, TS, and PJC conceived the study. NS and ALS managed the project. ALS performed and analyzed the sleep studies. UEE performed the MRS studies. CM helped with recruitment. $\mathrm{MMH}$ and MAG developed the reward and punishment task. CJH designed the emotional processing studies that were performed and analyzed by NS. NS compiled and analyzed the data with input from all authors. NS and GCC wrote the manuscript with input from all the authors.

\section{REFERENCES}

Agam G, Bersudsky Y, Berry GT, Moechars D, Lavi-Avnon Y, Belmaker RH (2009). Knockout mice in understanding the mechanism of action of lithium. Biochem Soc Trans 37: $1121-1125$.

Atack JR, Cook SM, Watt AP, Fletcher SR, Ragan CI (1993). In vitro and in vivo inhibition of inositol monophosphatase by the bisphosphonate L-690,330. J Neurochem 60: 652-658.

BALANCE investigators and collaborators, Geddes JR, Goodwin GM, Rendell J, Azorin J-M, Cipriani A et al (2010). Lithium plus valproate combination therapy versus monotherapy for relapse prevention in bipolar I disorder (BALANCE): a randomised open-label trial. Lancet 375: 385-395.

Berridge MJ, Downes CP, Hanley MR (1989). Neural and developmental actions of lithium: a unifying hypothesis. Cell 59: 411-419.

Bond A, Lader M (1974). The use of analogue scales in rating subjective feelings. Br J Med Psychol 47: 211-218.

Brant-Zawadzki M, Gillan GD, Nitz WR (1992). MP RAGE: a threedimensional, T1-weighted, gradient-echo sequence-initial experience in the brain. Radiology 182: 769-775.

Cavalla D (2009). APT drug R\&D: the right active ingredient in the right presentation for the right therapeutic use. Nat Rev Drug Discov 8: 849-853.

Conn PJ, Roth BL (2008). Opportunities and challenges of psychiatric drug discovery: roles for scientists in academic, industry, and government settings. Neuropsychopharmacology 33: 2048-2060.

Davanzo P, Yue K, Thomas MA, Belin T, Mintz J, Venkatraman TN et al (2003). Proton magnetic resonance spectroscopy of bipolar disorder versus intermittent explosive disorder in children and adolescents. Am J Psychiatry 160: 1442-1452.

Deelchand DK, Adanyeguh IM, Emir UE, Nguyen T-M, Valabregue R, Henry P-G et al (2014). Two-site reproducibility of cerebellar and brainstem neurochemical profiles with shortecho, single-voxel MRS at 3T. Magn Reson Med 73: 1718-1725.
Fischer H, Hilboll G, Romer A, Terlinden R. The Use of Highly Enriched 77Se in Metabolic Studies of Ebselen in Man - An NMR Invesigation. Springer Verlag: Berlin, 1989.

Fischer H, Terlinden R, Löhr JP, Römer A (1988). A novel biologically active selenoorganic compound. VIII. Biotransformation of ebselen. Xenobiotica 18: 1347-1359.

Friston KJ, Sharpley AL, Solomon RA, Cowen PJ (1989). Lithium increases slow wave sleep: possible mediation by brain 5 -HT2 receptors? Psychopharmacology (Berl) 98: 139-140.

Harmer CJ, Cowen PJ, Goodwin GM (2011). Efficacy markers in depression. J Psychopharmacol 25: 1148-1158.

Harmer CJ, Grayson L, Goodwin GM (2002). Enhanced recognition of disgust in bipolar illness. Biol Psychiatry 51: 298-304.

Harmer CJ, O'Sullivan U, Favaron E, Massey-Chase R, Ayres R, Reinecke A et al (2009). Effect of acute antidepressant administration on negative affective bias in depressed patients. Am J Psychiatry 166: 1178-1184.

Harmer CJ, Shelley NC, Cowen PJ, Goodwin GM (2004). Increased positive versus negative affective perception and memory in healthy volunteers following selective serotonin and norepinephrine reuptake inhibition. Am J Psychiatry 161: 1256-1263.

Häussinger D, Laubenberger J, vom Dahl S, Ernst T, Bayer S, Langer $M$ et al (1994). Proton magnetic resonance spectroscopy studies on human brain myo-inositol in hypo-osmolarity and hepatic encephalopathy. Gastroenterology 107: 1475-1480.

Lang PJ, Greenwald MK, Bradley MM, Hamm AO (1993). Looking at pictures: affective, facial, visceral, and behavioral reactions. Psychophysiology 30: 261-273.

Lopez AD, Mathers CD, Ezzati M, Jamison DT, Murray CJL (2006). Global and regional burden of disease and risk factors, 2001: systematic analysis of population health data. Lancet 367: 1747-1757.

Lynch E, Kil J (2009). Development of ebselen, a glutathione peroxidase mimic, for the prevention and treatment of noiseinduced hearing loss. Semin Hear 30: 047-055.

McKnight RF, Adida M, Budge K, Stockton S, Goodwin GM, Geddes JR (2012). Lithium toxicity profile: a systematic review and meta-analysis. Lancet 379: 721-728.

Moore GJ, Bebchuk JM, Parrish JK, Faulk MW, Arfken CL, Strahl-Bevacqua J et al (1999). Temporal dissociation between lithium-induced changes in frontal lobe myo-inositol and clinical response in manic-depressive illness. Am J Psychiatry 156: 1902-1908.

Motulsky H, Christopoulos A. Global models. Fitting Models to Biological Data Using Linear and Nonlinear Regression: A Practical Guide to Curve Fitting. GraphPad Software Inc.: San Diego, CA, 2003.

Murphy SE, Downham C, Cowen PJ, Harmer CJ (2008). Direct effects of diazepam on emotional processing in healthy volunteers. Psychopharmacology (Berl) 199: 503-513.

Natt O, Bezkorovaynyy V, Michaelis T, Frahm J (2005). Use of phased array coils for a determination of absolute metabolite concentrations. Magn Reson Med 53: 3-8.

O'Brien WT, Klein PS (2009). Validating GSK3 as an in vivo target of lithium action. Biochem Soc Trans 37: 1133-1138.

Parnham MJ, Sies H (2013). The early research and development of ebselen. Biochem Pharmacol 86: 1248-1253.

Parrott AC, Hindmarch I (1980). The Leeds sleep evaluation questionnaire in psychopharmacological investigationsa review. Psychopharmacology (Berl) 71: 173-179.

Patel NC, Cecil KM, Strakowski SM, Adler CM, DelBello MP (2008). Neurochemical Alterations in Adolescent Bipolar Depression: A Proton Magnetic Resonance Spectroscopy Pilot Study of the Prefrontal Cortex. J Child Adolesc Psychopharmacol 18: 623-627.

Perry BD. Memories of Fear. In: J Goodwin, R Attias (eds). Splintered Reflections: Images of the Body in Trauma. Basic Books: Washington, DC, 1999, pp 9-38. 
Phillips ML, Swartz HA (2014). A critical appraisal of neuroimaging studies of bipolar disorder: toward a new conceptualization of underlying neural circuitry and a road map for future research. Am J Psychiatry 171: 829-843.

Provencher SW (1993). Estimation of metabolite concentrations from localized in vivo proton NMR spectra. Magn Reson Med 30: 672-679.

Quiroz JA, Gould TD, Manji HK (2004). Molecular effects of lithium. Mol Interv 4: 259-272.

Rey A (1964). L'examen clinique en psychologie. Presses Universitaires de France: Paris.

Rock PL, Goodwin GM, Harmer CJ (2010). The common adolescent bipolar phenotype shows positive biases in emotional processing. Bipolar Disord 12: 606-615.

Shah S, Kellman P, Greiser A, Weale P, Zuehlsdorff S, Jerecic R (2009). Rapid Fieldmap Estimation for Cardiac Shimming. In: Proceedings of the 17th Scientific Meeting, International Society for Magnetic Resonance in Medicine. Honolulu: ISMRM; 2009. Abstract nr 565.

Sharma R, Venkatasubramanian PN, Bárány $M$, Davis JM (1992). Proton magnetic resonance spectroscopy of the brain in schizophrenic and affective patients. Schizophr Res 8: 43-49.

Sharpley AL, Elliott JM, Attenburrow MJ, Cowen PJ (1994). Slow wave sleep in humans: role of 5-HT2A and 5-HT2C receptors. Neuropharmacology 33: 467-471.

Silverstone PH, McGrath BM (2009). Lithium and valproate and their possible effects on the myo-inositol second messenger system in healthy volunteers and bipolar patients. Int Rev Psychiatry 21: 414-423.
Silverstone PH, McGrath BM, Kim H (2005). Bipolar disorder and myo-inositol: a review of the magnetic resonance spectroscopy findings. Bipolar Disord 7: 1-10.

Singh N, Halliday AC, Thomas JM, Kuznetsova OV, Baldwin R, Woon ECY et al (2013). A safe lithium mimetic for bipolar disorder. Nat Commun 4: 1332.

Smith SM (2002). Fast robust automated brain extraction. Hum Brain Mapp 17: 143-155.

Spielberger CD, Gorsuch RL, Lushene RE. Manual for the State-Trait Anxiety Inventory. Consulting Psychologists Press: Palo Alto, CA, 1970.

Von Zerssen D. Clinical Self-Rating Scales (CSRS) of the Munich Psychiatric Information System (PSYCHIS München). In: Sartorius N, Ban TA (eds). Assessment of Depression. Springer: Berlin, Heidelberg, 1986, pp 270-303.

Watson D, Clark LA, Tellegen A (1988). Development and validation of brief measures of positive and negative affect: The PANAS scales. J Pers Soc Psychol 54: 1063-1070.

Whitton AE, Treadway MT, Pizzagalli DA (2015). Reward processing dysfunction in major depression, bipolar disorder and schizophrenia. Curr Opin Psychiatry 28: 7-12.

Yamaguchi T, Sano K, Takakura K, Saito I, Shinohara Y, Asano T et al (1998). Ebselen in acute ischemic stroke: a placebocontrolled, double-blind clinical trial. Ebselen Study Group. Stroke J Cereb Circ 29: 12-17.

Zarate CA, Manji HK (2009). Protein kinase C inhibitors: rationale for use and potential in the treatment of bipolar disorder. CNS Drugs 23: $569-582$.

Zhang H, Liu J, Zhu Z, Li H (2011). An automated and simple method for brain MR image extraction. Biomed Eng Online 10: 81.

Supplementary Information accompanies the paper on the Neuropsychopharmacology website (http://www.nature.com/npp) 